\title{
Refinement of Inductor Models via a Subproblem Finite Element Method
}

\author{
Patrick Dular ${ }^{1,2}$, Mauricio V. Ferreira da Luz ${ }^{3}$, Patrick Kuo-Peng ${ }^{3}$, Ruth V. Sabariego ${ }^{1}$, \\ Laurent Krähenbühl $^{4}$ and Christophe Geuzaine ${ }^{1}$ \\ ${ }^{1}$ University of Liège, Dept. of Electrical Engineering and Computer Science, ACE, B-4000 Liège, Belgium \\ ${ }^{2}$ F.R.S.-FNRS, Fonds de la Recherche Scientifique, Belgium \\ ${ }^{3}$ GRUCAD/EEL/UFSC, Po. Box 476, 88040-970 Florianópolis, Santa Catarina, Brazil \\ ${ }^{4}$ Université de Lyon, Ampère (CNRS UMR5005), École Centrale de Lyon, F-69134 Écully Cedex, France
}

\begin{abstract}
A subproblem finite element method is developed for refining the inductor models in magnetic circuits. It applies electrokinetic, magnetostatic and magnetodynamic models on progressively more complicated geometries and different components of the solution, supported by different meshes. It allows an efficient and robust analyze of magnetic circuits in any frequency range, with an accurate calculation of Joule losses and forces in inductors.
\end{abstract}

\section{INTRODUCTION}

The finite element (FE) modeling of inductors, i.e. the active parts of magnetic circuits, can be tackled at various levels of precision. Their geometry as well as the distribution of the current they carry may be simplified to lighten the computational efforts, especially at the preliminary stages of a design. Then, refinements have to be performed to ensure a required accuracy, that is generally problem dependent. Progressive refinements can be done from stranded to massive inductors, and from static to dynamic excitations, to accurately render skin and proximity effects, i.e. the non-uniformly distributed fields and current densities. In particular, an accurate determination of Joule losses and force densities in inductors lies on finely calculated local fields.

A method is developed herein for sequencing FE models of inductors, i.e. electrokinetic, magnetostatic and magnetodynamic models applied on progressively more complicated geometries. At each step of the refinement, a correction to be added to the previous solution is calculated and its usefulness is evaluated via its relative contribution. The proposed method naturally fits into the subproblem approach developed by the authors in [1] for other refinements. The intensive re-use of subproblem solutions is very efficient for parametric analyses. A different mesh is used for each subproblem and the successive sources are obtained by means of Galerkin projections between them. These have to be properly discretized to assure the conformity of all the sequenced FE weak formulations.

\section{SEQUENCE OF INDUCTOR MODELS}

The inductor modeling starts with an electrokinetic model, possibly applied on a simplified geometry, e.g. a zerothickness (shell) inductor. For a massive inductor, this model asks for a FE calculation, whereas a stranded inductor can be given an a priori known uniform current density. The resulting current density becomes the source of a magnetostatic model applied on the same inductor geometry and surrounding domain including or not all the material and other inductor regions. These can be further added as perturbations [1].

These preliminary steps are proposed herein before any magnetodynamic computation and estimating the necessity of a refinement. Indeed, in some cases, e.g. for low frequency ex-

This work was supported by the F.R.S.-FNRS (Belgium), the CNPq (Brazil), the Belgian Science Policy (IAP P6/21) and the Walloon Region. citations or significant distances between the inductors and the other regions, a good accuracy can be already obtained. Only the coupling of several inductors via their lumped inductances has to be considered, with no need of inductive coupling at the local FE level. In such conditions, solving the magnetodynamic problem in only one step would usually result in an illconditioned matrix system [2], which is avoided here. However, for high frequencies, conductivities and permeabilities, and short distances between the regions, the current densities are affected by skin and proximity effects, that are to be calculated via refinements of the static distributions.

For massive inductors (Fig. 1), the conductivity , initially neglected with regard to skin and proximity effects, is then considered. This defines a change of conductivity from 0 to and thus a modified ohm law for the refinement subproblem [1]: $\boldsymbol{j}_{2}=\boldsymbol{e}_{2}+\boldsymbol{e}_{1}$, with $\boldsymbol{j}_{2}$ and $\boldsymbol{e}_{2}$ the unknown correction current density and electric field, and $\boldsymbol{e}_{1}$ the source electric field induced by the time variation of the magnetic flux density $\boldsymbol{b}_{1}$ given by the static solution. A possible split of skin and proximity effects can be done, which adds refinement steps. As a complementary approach, a change from an infinite conductivity, thus a perfect conductor, to the actual conductivity was done in [3]. A refinement from a shell inductor to a massive inductor can be done via a simultaneous change of interface condition and of the so-added volume region [1]. In practice, this leads to a clear view of the effect on leakage flux and skin and proximity effects. Each refinement of each inductor leads to a correction of its inner and outer fields as well as of its circuit relation, i.e. its resistance and inductance. All the refinement steps will be detailed, illustrated and validated in the extended paper, with particular applications for 3-D inductors in induction heating and microcoils.
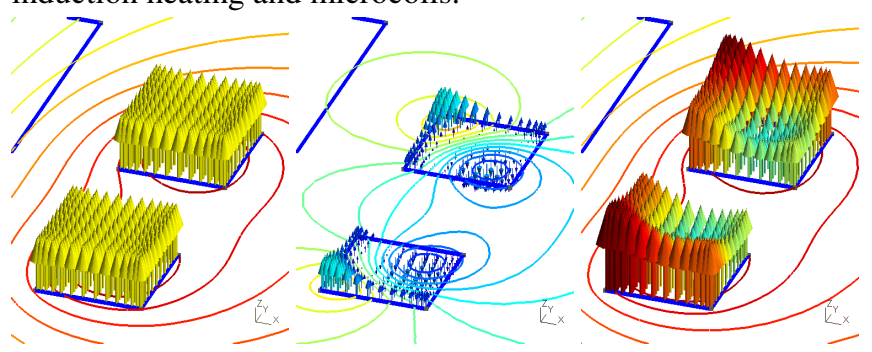

Fig. 1. Field lines and current density for an inductor (cross-section): static state (left), magnetodynamic correction (middle) and total solution (right).

\section{REFERENCES}

[1] P. Dular, R. V. Sabariego, M. V. Ferreira da Luz, P. Kuo-Peng and L. Krähenbühl, "Perturbation Finite Element Method for Magnetic Model Refinement of Air Gaps and Leakage Fluxes," IEEE Trans. Magn., Vol.45, No.3, pp. 1400-1403, 2009.

[2] R. Hiptmair, F. Kramer and J. Ostrowski, "A robust Maxwell formulation for all frequencies," IEEE Trans. Magn., Vol. 44, No. 6, pp. 682-685, 2008.

[3] P. Dular, R. V. Sabariego, J. Gyselinck, L. Krähenbühl, "Sub-domain finite element method for efficiently considering strong skin and proximity effects," COMPEL, Vol.26, No.4, pp.974-985, 2007. 